\title{
Gut Micro biome, Multiple Sclerosis, and Cancer
}

\author{
Robert Skopec* \\ Researcher-analyst, Dubnik, Slovakia

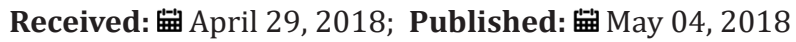 \\ *Corresponding author: Robert Skopec, Researcher-analyst, Dubnik, Slovakia, Email: zxcbnvm7@gmail.com
}

\begin{abstract}
Thanks to their influence on the owner's gut micro biome, dogs may be beneficial in reducing cardiovascular, diabetes, multiple sclerosis and cancer risk by providing a non-human form of social support and increasing physical activity. Ownership of hunting dog breeds was associated with a decreased risk of CVD, and ownership of all purebred breeds was associated with lower risk of all-cause mortality. Dog ownership has also been associated with elevated parasympathetic and diminished sympathetic nervous system activity, lower reactivity to stress, and faster recovery of blood pressure following stressful activity. We provide evidence that supports epsilon toxin's ability to cause BBB permeability and show that epsilon toxin kills the brain's myelin producing cells, oligodendrocytes; the same cells die in multiple sclerosis (MS) lesions. A new study has identified how the environment surrounding a tumor can stimulate metastatic behavior in the individual cancer cells. Researchers found that the tumor cells activate a particular set of genes and begin form blood vessel-like structure when they are confined in densely packed environment
\end{abstract}

Keywords: Gut Micro Biome; Dog Ownership; Multiple Sclerosis; Cancer; Dyslipidemia; Hypertension and Diabetes; Reduced CVD Risk; Mortality; UCSF: University of California, San Francisco;

Abbreviations: MS: Multiple Sclerosis; CVD: Cardiovascular Disease; BBB: Blood Brain Barrier; UCSD: University of California San Diego; CINP: Collagen-Induced Network Phenotype

\section{Introduction}

Cardiovascular disease (CVD) is the leading cause of death worldwide, accounting for $45 \%$ of all deaths ( $>4$ million) in Europe in 2016. Dogs may be beneficial in reducing cardiovascular risk by providing a non-human form of social support and increasing physical activity. Dog ownership has been reported to be associated with alleviation of social isolation and improved perception of wellbeing, particularly in single persons and the elderly. A metaanalysis of eleven studies found that dog owners walked more and were more physically active than non-owners. Two studies assessing changes in physical activity after acquisition of a dog or other pet found increased self-reported recreational walking. A one study showed that dog ownership also supports the maintenance of physical activity during poor weather.

\section{Dog Ownership is Associated with Decreased CVD Risk}

Previous findings on dog ownership and the risk of CVD are conflicting. In individuals without CVD, dog ownership has been reportedasinverselyassociated with theprevalenceofcardiovascular risk factors, such as dyslipidemia, hypertension and diabetes,

but other studies found absent or inconclusive associations. In patients with coronary artery disease, dog ownership is reportedly associated with improved survival. Although the American Heart Association issued a Scientific Statement in 2013 concluding that "[...] dog ownership is probably associated with decreased CVD risk [...]" and that this effect "[...] may be causal [...]", earlier studies have had several limitations, including lack of power, low responder rates and incomplete adjustment for potential confounders. We try to clarify the association of dog ownership with CVD and mortality by the help of published over 3.4 million Swedish adults followed through nationwide register linkage over a 12-year period. In this register-based nationwide prospective study including 3,432,153 individuals, dog ownership was associated with a lower risk of incident cardiovascular disease in single-person households and with lower cardiovascular and all-cause mortality in the general population. Ownership of hunting dog breeds was associated with a decreased risk of CVD, and ownership of all purebred breeds was associated with lower risk of all-cause mortality. Although further investigation in the twin cohort did not show any association 
between dog ownership and CVD and mortality likely due to the smaller sample size, additional adjustment for detailed lifestyle and socioeconomic factors only marginally altered these estimates.

There might be direct effects of dog ownership on health outcomes. One mechanism by which dog ownership could reduce CVD risk and mortality is by alleviating psychological stress factors, such as social isolation, depression and loneliness - all reportedly lower in dog owners. These factors have been linked to increased risk of coronary heart disease, cardiovascular death and all-cause mortality. Dog ownership has also been associated with elevated parasympathetic and diminished sympathetic nervous system activity, lower reactivity to stress, and faster recovery of blood pressure following stressful activity. Apart from the social support, it has consistently been shown that dog owners achieve more physical activity and spend more time engaged in outdoor activities. Individuals in single households benefitted most from dog ownership regarding protection from CVD. A study on the psychological effects of dog ownership suggested that ownership benefits single persons more than married individuals. Moreover, single dog-owners were shown to walk their dog more often than individuals in multiple-person households, and in general, it is plausible that not all members of a multiple-person interact with the dog as much as a single owner. In both multiple- and single person household strata, can be found lower hazard ratios for dog ownership on all-cause mortality and cardiovascular mortality than on incident CVD. This discrepancy may be explained by less severe disease at hospital presentation in dog-owners, similar to the effect described in physically active persons. Alternatively, owning a dog may improve rehabilitation after an incident disease event by acting as motivation and support to mobilize for walks again. In conclusion, in a nationwide population based study with 12 years of follow-up there was shown that dog ownership is associated with lower risk of cardiovascular disease in single households and with a reduced risk of cardiovascular and all-cause death in the general population Cline [1]; Christian et al. [2]; Mubanga et al. [3].

\section{Dog Ownership Linked to Lower Mortality}

A new study by Tove Fall and colleagues shows that dog ownership had a lower risk of death. A team of Swedish researchers have used national registries of more than 3.4 million Swedes aged 40 to 80 to study the association between dog ownership and cardiovascular health. Their study shows that dog owners had a lower risk of death due to cardiovascular disease or to other causes during the 12-year follow-up.

A total of more than 3.4 million individuals without any prior cardiovascular disease in 2001 were included in the researchers' study linking together seven different national data sources, including two dog ownership registers. The goal was to determine whether dog owners had a different risk of cardiovascular disease and death than non-dog owners. A very interesting finding in their study was that dog ownership was especially prominent as a protective factor in persons living alone, which is a group reported previously to be at higher risk of cardiovascular disease and death than those living in a multi-person household. Perhaps a dog may stand in as an important family member in the single households. The results showed that single dog owners had a 33\% reduction in risk of death and $11 \%$ reduction in risk of myocardial infarction during follow-up compared to single non-owners. Another interesting finding was that owners to dogs from breed groups originally bred for hunting were most protected, says Mwenya Mubanga, lead junior author of the study and PhD student at the Department of Medical Sciences and the Science for Life laboratory, Uppsala University. These kinds of epidemiological studies look for associations in large populations but do not provide answers on whether and how dogs could protect from cardiovascular disease. Dog owners in general have a higher level of physical activity, which could be one explanation to the observed results. Other explanations include an increased well-being and social contacts or effects of the dog on the bacterial micro biome in the owner, says Tove Fall, senior author of the study and Associate Professor in Epidemiology at the Department of Medical Sciences and the Science for Life laboratory, Uppsala University. There might also be differences between owners and non-owners already before buying a dog, which could have influenced above results, such as those people choosing to get a dog tending to be more active and of better health. Thanks to the population-based design, results are generalisable to the Swedish population, and probably also to other European populations with similar culture regarding dog ownership, says Tove Fall. The study was conducted by researchers at Uppsala University, Karolinska Institute, Stanford University and the Swedish University of Agricultural Sciences Mubanga et al. [3].

\section{Dog-Owners Live Longer}

Swedish scientists have found that owning a dog can lower a persons' risk of mortality, in study published in Scientific Report/ (Nature). Researchers examined data relating to Swedes aged between 40 and 80, who in 2001 (when registration of dog ownership was first made obligatory had no history of cardiovascular disease. Over the next 12 years, dog owners had a lower risk of death due to cardiovascular disease or other causes than those without a dog, according to the study's results. The effect was particularly pronounced among people who lived alone, with dog-owners in single households having a 33percent reduction in mortality and an 11 percent reduction of heart attack compared to people living alone who did not own a dog. This was one of the most interesting findings: the researchers see that dogs have a larger impact in households without other humans, told Tove Fall, the study's senior author and an associate professor at Uppsala University to the journal The Local. This could be because if you're the sole dog owner, you get more physical activity and it could also show that dogs help alleviate stress from loneliness. The finding demonstrates the important role dogs play in most developed countries, such as USA and Europe, where a high proportion of the population, especially among the elderly, live alone. 
In these most developed countries people often are not so good at talking to other people, and a dog could help with that: it's an ice-breaker for interaction. There are several possible reasons for the positive effects dogs appear to have on their owners' health. One potential reason is the effect on micro biomes. Lots of experts think that nowadays we have poor diversity in micro biome (see also MS!), and it might be that dogs have a positive effect on this by bringing in dirt and bacteria, T. Fall said. The effect also varies across different breed groups, according to the study's findings, with owners of larger, more active breeds drawing the most health benefits from their canine pals. The breeds with strongest positive effects on owners' health were the retriever group, including golden retrievers and Labradors, and pointing breeds.

I hope that there will be a higher acceptance of dogs in society, said T. Fall. It's not so easy to combine dogs with an active work life and they aren't always allowed in places, so the study could help us to understand that dogs are really important for their owners, and for example be more understanding if someone's dog passes away Edwards [4].

\section{Bacterial Toxin a Potential Triggers Multiple Sclerosis}

Researchers from Weill Cornell Medical College have added to the growing body of evidence that multiple sclerosis (MS) may be triggered by a toxin produced by common food borne bacteria. Multiple sclerosis is an inflammatory disease of the central nervous system characterized by Blood Brain Barrier (BBB) permeability and demyelization, a process in which the insulating myelin sheaths of neurons are damaged. The disease is thought to be triggered in a genetically susceptible individual by a combination of one or more environmental factors. We provide evidence that supports epsilon toxin's ability to cause BBB permeability and show that epsilon toxin kills the brain's myelin producing cells, oligodendrocytes, the same cells die in MS lesions, says Jennifer Linden of Weill Cornell Medical College. They also show that epsilon toxin targets other cell types associated with MS inflammation such as the retinal vascular and meningeal cells. Epsilon toxin may be responsible for triggering MS. Epsilon toxin is produced by certain strains of Clostridium perfringens, a spore-forming bacterium that is one of the most common causes of food borne illness in the United States. The U. S. Centers for Disease Control and Prevention estimates that non-epsilon producing $C$. perfringens strains cause nearly a million cases of food borne illness each year.

Previous studies have suggested that $C$. perfringens and in particular epsilon toxin, may play role in triggering MS. J. Linden and her colleagues discovered also C. perfringens type B (a strain that is not known to infect humans and produces the epsilon toxin) in a 21-year old women who was experiencing a flare-up of her MS. To further test their hypothesis J. Linden and her colleagues studied the behavior of the toxin in mice, specifically which cells it targeted. They discovered that the toxin did target the brain cells associated with MS pathology.
Originally we only thought that epsilon toxin would target the brain endothelium and oligodendrocytes, we just happened to notice that it also bound to and killed meningeal cells. This was exciting because it provides a possible explanation for meningeal inflammation and subpial cortical lesions exclusively observed in MS patients, says J. Linden. They also tested samples of local foods for the presence of $C$. perfringens and athe toxin gene. Of the 37 food samples, $13,5 \%$ were positive for bacteria and 2,7\% were positive for the epsilon toxin gene. J. Linden says these findings are important, because if it can be confirmed that epsilon toxin is indeed a trigger of MS, development of a neutralizing antibody or vaccine directed against epsilon toxin might stop the progression of the disease or prevent it from developing The American Society for Microbiology, BBC NEWS [5,6].

\section{Gut Bacteria May Cause Multiple Sclerosis}

Certain types of bacteria in the gut may play role in the progression of multiple sclerosis (MS). The research, the study authors believe, could lead to new ways to treat multiple sclerosis, an autoimmune neurodegenerative disease that affects about 2.5 million people worldwide. The gut micro biome is very malleable, said Sergio Baranzini, study senior author. You could relatively easily change it in an adult, who has MS or is susceptible - something you cannot do with their genetics. This is not a magical approach, but it is hopeful. MS occurs when the immune system attacks the insulation, or myelin, around nerve cells. This can lead to vision loss, weakness, problems with coordination and balance and, in some cases, paralysis. The study included 71 patients and control group of 71 healthy people. Specific species of gut bacteria, or microbes, were more common in people with MS. When these species of gut microbes were transplanted into mice, the microbes affected their immune system.

The field has been very successful in identifying genes associated with susceptibility to MS, but, we could be not satisfied with the amount of risk that we'll be able to explain with just genetics, said S. Baranzini, a professor of neurology at the University of California, San Francisco (UCSF). Even identical twins, who share the same genetic inheritance, only share an MS diagnosis about 35 percent of the time. It's clear the genome is important, but environmental factors, such as diet, smoking and surroundings must play also a major role. Previous studies have shown that gut microbiomes have a direct influence on the immune system, so the UCSF researchers decided to investigate what role these microbes might play in MS. The findings were published online Sept. 11 in the journal Proceedings of the National Academy of Sciences.

A second study in the same journal issue also found that transplanting certain types of gut bacteria from MS patients into mice affected the animals immune system. Two different groups, using two separate cohorts of patients and controls, and two distinct mouse models of the disease, saw very similar results. This is very promising evidence that we're on the right track, said Egle Cekanaviciute, a UCSF postdoctoral researcher. To be clear, they 
don't think the microbiome is the only trigger of MS. But it looks like these microbes could be making the disease progression worse or better-pushing someone with genetic predisposition across the treshold into disease or keeping them safe, E. Cekanaviciute explained. Possible treatments includes diet changes or drugs based on microbial byproducts, according to the researchers. (The U.S. National Institute of Neurological Disorders and Stroke on multiple sclerosis, 2017)

\section{Specific Tumor Environments Stimulates Cancer Cells to Metastasize}

A new study, conducted by a team of researchers from the University of California, San Diego (UCSD) [7], has identified how the environment surrounding a tumor can stimulate metastatic behavior in the individual cancer cells. Researchers found that the tumor cells activate a particular set of genes and begin form blood vessel-like structure when they are confined in densely packed environment. These structures were observed earlier by physicians in the hospital in a phenomenon known as vascular mimicry, which is linked to some of the most aggressive types of cancers. But the cause of this transformation was unknown to them. $90 \%$ of cancer deaths are caused by the metastatic spread of cancer cells from one site of the body to another. The set of genes discovered by researchers, known as a gene module, was capable of predicting the life expectancy of the patient and whether tumors will metastasize across nine cancer types, which includes kidney, pancreatic, lung, and breast cancers. This gene module could be involved in determining whether patients are suffering from an aggressive cancer type and inform the decisions that patients and their oncologists make when choosing particular therapies [8-10].

The researchers made their observations by positioning the malignant cells in a custom 3D collagen matrix built. The identified that the cells turned into resembling blood vessels when surrounded by the matrix made of small pores and short fibers (as opposed to alrge pores and long fibers) and that this transformation was independent of other physical characteristics of the matrix like stifness. Daniel Ortiz Velez, first author of the study, stated that researchers thought that confining cells into more constrained type of environment would stop their spread, but opposite happened. Researchers further added that this type of behavior was not exhibited by the cells in traditional petri dishes. Stephanie Fraley, leader of the study and professor of bioenginiering at the UCSD stated that it's critical to have the cells surrounded by a 3D environment that mimics what happens in the human body. In addition, researchers indentified that the behavior of the cell is caused by a particular gene module,which they called collageninduced network phenotype (CINP). Confining these cells into a constrained environment rewrites their gene expression. S. Fraley indicated that this phenomenon is almost similar to the matrix encoding the gene module. Furthermore, researchers looked for this gene module in a range of human cancer gene expression and histology databasess, which comprise details of the tissue microscopic structure. The presence of the gene module was a strong predictor of whether the tumor cells seemed to metastasize aggressively, after accounting other factors, like the patient's age. This makes sense as the channels constructed by the malignant cells permits blood to flow to tumors without coagulating, as well as aiding the collectio of nutrients. This also makes it simple for tumor cells to spread through the patient's blood. Other researchers have indicated that when cancer cells are connected, it enhances their chances of spreading to distant locations in the body.

\section{Acknowledgement}

The author gratefully acknowledge the assistance of Dr.Marta Ballova, Ing. Konrad Balla, Livuska Ballova, and Ing Jozef Balla.

\section{Refernces}

1. Cline KM (2010) Psychological effects of dog ownership: role strain, role enhancement, and depression. The Journal of social psychology 150(2): 117-131.

2. Christian HE, Westgarth C, Bauman A, Richards EA, Rhodes RE, et al. (2013) Dog ownership and physical activity: a review of the evidence. Journal of physical activity \& health 10(5): 750-759.

3. Mubanga M, et al. (2017) Dog ownership and the risk of cardiovascular disease and death-a nationwide cohort study. Scientific Reports 7.

4. Edwards C (2017) Dog-owners live longer, say Swedish scientists.

5. BBC NEWS: http://bbc.uk/news/health-25925658?print=true.

6. https://doi.org/10.1080/00224540903368533 (2010).

7. (2017) Researchers discover specific tumor environment that triggers cells to metastasize. University of California -San Diego, USA.

8. doi:10.1038/s4198-017-16118-6.

9. The ASM: https://www.asm.org/index.php/asm-newsroom2/92news-room/press-releases

10. (2017) The U.S. National Institute of Neurological Disorders and Stroke on multiple sclerosis. 
This work is licensed under Creative Commons Attribution 4.0 License

Submission Link:

Submit Article

DOI: $10.32474 /$ DDIPIJ.2018.01.000113

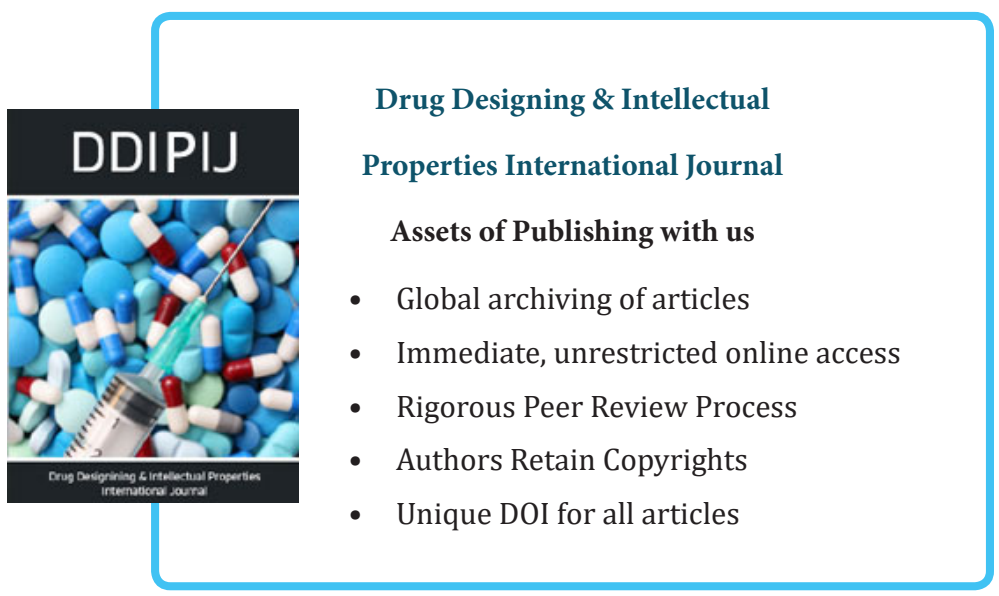

